\title{
EFECTO DE LA CONCENTRACIÓN DE POLEN APÍCOLA EN LAS PROPIEDADES REOLÓGICAS, COLORIMÉTRICAS Y FISICOQUIIMICAS DEL JUGO DE NARANJA
}

Recibido: julio del 2018

Aceptado: marzo del 2019

Rigoberto Villa Ramírez ${ }^{1}$, Tatiana Sánchez Ante², Lina Arbeláez Arias ${ }^{3}$

\section{Resumen}

Al tener en cuenta los beneficios del jugo de naranja y el consumo de polen para el organismo, el propósito de este trabajo fue la estandarización de un jugo de naranja enriquecido con polen, para ello se elaboraron dos tipos de jugo con diferentes concentraciones de polen, P1\% y P5\%. Se evaluaron las coordenadas de color $\left(L^{*}, a^{*}\right.$ y $\left.\mathrm{b}^{\star}\right)$, el comportamiento reológico para determinar el comportamiento al flujo del jugo y las características fisicoquímicas. Los parámetros colorimétricos $\mathrm{L}^{*}, \mathrm{a}^{*} \mathrm{y} \mathrm{b}^{*}$ del sistema CIELab de color de un jugo comercial con relación al jugo enriquecido con polen aumentó para los valores de $\mathrm{L}^{*} \mathrm{y} \mathrm{a}^{*}$, debido a mayor contribución de la luminosidad y del color rojo con un aumento en la concentración de polen. El comportamiento de flujo evidenció un comportamiento newtoniano para el jugo al $1 \%$, el cual pasa a ser un fluido no newtoniano con un aumento en la concentración de jugo. El análisis fisicoquímico, por medida de ${ }^{\circ} \mathrm{Brix}, \mathrm{pH}$ y acidez titulable, permite concluir que los tratamientos analizados cumplen con lo especificado en la norma técnica colombiana (NTC) 5468 Jugo (zumo), pulpa, néctar de frutas y sus concentrados.

Palabras clave: abejas, bebida, cítricos, producto, vitaminas.

${ }^{1}$ Magíster en Biología Vegetal. Universidad del Quindío. Correo: rivilla@uniquindio.edu.co

${ }^{2}$ Magíster en Química I. Universidad del Quindío. Correo: Itsanchez@uniquindio.edu.co

${ }^{3}$ Magíster en Biología Vegetal. Universidad del Quindío. Correo: linama@uniquindio.edu.co 


\section{EFFECTS OF POLLEN CONCENTRATION IN THE RHELOGICAL, COLORIMETRIC, PHYSICAL AND CHEMICAL PROPERTIES OF ORANGE JUICE}

Rigoberto Villa Ramírez ${ }^{1}$, Tatiana Sánchez Ante², Lina Arbeláez Arias ${ }^{3}$

\section{Abstract}

Taking into account the benefits of drinking orange juice and pollen, we decided to propose a research to standardize a type of orange juice enriched with pollen. Thus, we made two kinds of juice with different concentrations of pollen: $\mathrm{P} 1 \%$ and $\mathrm{P} 5 \%$. We evaluated its color coordinates $\left(\mathrm{L}^{*}, \mathrm{a}^{\star} \mathrm{y} \mathrm{b}^{*}\right)$, its rheological behavior and its physical and chemical characteristics. Colorimetric criterion $\mathrm{L}^{*}, \mathrm{a}^{*} \mathrm{y} \mathrm{b}^{*}$ of the CIELab system for the color of commercial juice, in comparison to the enriched juice with pollen, increased for the $\mathrm{L}^{*}$ and $\mathrm{a}^{*}$ values, given the bigger contribution to luminosity and red color that brings the pollen. Rheological behavior showed a Newtonian behavior for the juice with $1 \%$ concentration, becoming a non-Newtonian liquid after the concentration. Physical and chemical analysis, through measurements of Brix, $\mathrm{pH}$ and titrateable acidity ratios, allows to conclude that the analyzed treatments comply with the specifications of the 5468 Colombian Technical Norm (NTC in Spanish): juice, pulp, fruit nectar and its concentrates. 


\section{EFEITO DA CONCENTRAÇÃO DE PÓLEN APÍCOLA NAS PROPRIEDADES REOLÓGICAS, COLORIMÉTRICASE FÍSICO-QUÍMICAS DO SUCO DE LARANJA}

Rigoberto Villa Ramírez ${ }^{1}$, Tatiana Sánchez Ante², Lina Arbeláez Arias ${ }^{3}$

\section{Resumo}

Ao considerar os benefícios do suco de laranja e o consumo de pólen para o organismo, o objetivo deste trabalho foi a padronização de um suco de laranja enriquecido com pólen; para isso, foram elaborados dois tipos de suco com diferentes concentrações de pólen: P1 \% e P5\%. Foram avaliadas as coordenadas de cor $\left(\mathrm{L}^{\star}, \mathrm{a}^{\star} \mathrm{e} \mathrm{b}^{*}\right)$, o comportamento reológico para determinar o comportamento no fluxo do suco e as características físico-químicas. Os parâmetros colorimétricos $\mathrm{L}^{\star}, \mathrm{a}^{\star} \mathrm{e}$ $\mathrm{b}^{*}$ do sistema CIELab de cor de um suco comercial em comparação com o enriquecido com pólen aumentaram para os valores de $\mathrm{L}^{*} \mathrm{e}$ $\mathrm{a}^{*}$, devido à maior contribuição da luminosidade e da cor vermelha com o aumento na concentração de pólen. O comportamento de fluxo evidenciou um comportamento newtoniano para o suco a $1 \%$, o que passa a ser um fluido não newtoniano com um aumento na concentração de suco. A análise físico-química, por medida de ${ }^{\circ}$ Brix, $\mathrm{pH}$ e acidez titulável, permite concluir que os tratamentos analisados cumprem com o especificado na norma técnica colombiana (NTC) 5468: suco (sumo), polpa, néctar de frutas e seus concentrados. 


\section{Introducción}

La naranja es el fruto del naranjo dulce con escaso valor calórico, aporta fibra soluble (pectina), ácido ascórbico, carotenoides con actividad provitamínica A (Algarra, 2017). Su importancia radica en su alto contenido de antioxidantes, sustancias capaces de bloquear el daño de los radicales libres, evitar el envejecimiento prematuro del organismo y prevenir enfermedades crónicas y degenerativas como el cáncer.

El polen de abeja es un conjunto de polvillos recolectados de las anteras de las flores y la combinación del néctar. Estas contienen proteínas de alto valor biológico, lípidos y vitaminas liposolubles (Gonzáles, 2011). Al polen se le atribuyen funciones benéficas al organismo humano, esto se debe a su alto contenido de sustancias nitrogenadas, aminoácidos esenciales, sales minerales, vitaminas entre otros componentes (Benedetti y Pieralli 1990).

En las últimas décadas, la tendencia al consumo de alimentos saludables ha llevado a la industria alimentaria hacia el desarrollo de alimentos balanceados. El consumo de productos naturales y saludables está aumentando, ya que los alimentos con procesos tradicionales van quedando de lado por su alto valor calórico (alto porcentaje de azúcar) y adición de conservantes (Guillermo, 2017).

Desde hace algunos años, la caracterización reológica de los productos alimentarios sigue una clara tendencia a desa-

rrollarse, debido a que el conocimiento adecuado de las propiedades reológicas tiene numerosas aplicaciones sobre las etapas del proceso de producción de diversos alimentos, tanto en el diseño de procesos y equipos, y en la evaluación sensorial como para el control de calidad, además de dar cuenta de la estructura del alimento (Barbosa-Cánovas, 1996). Al tener en cuenta las propiedades funcionales de los productos naturales y las características físicas de los alimentos, este trabajo tuvo como objetivo evaluar las propiedades colorimétricas, reológicas y fisicoquímicas de un jugo de naranja enriquecido con polen apícola en diferentes concentraciones.

\section{Materiales y métodos}

Las caracterizaciones del jugo de naranja con las diferentes concentraciones de polen fueron realizadas en el laboratorio de Pos cosecha de la Facultad de Ciencias Agroindustriales de la Universidad del Quindío por triplicado.

\section{Análisis fisicoquímicos}

Acidez titulable: se determinó la acidez titulable, expresada en porcentaje de ácido cítrico según la NTC 4623 de "Productos de frutas y verduras. Determinación de la acidez titulable".

pH: se realizó la medida del potencial de hidrógeno teniendo en cuenta la NTC 4592 "Productos de frutas y verduras. Determinación del pH”.

${ }^{\circ}$ Brix: el contenido de sólidos solubles se evaluó de acuerdo a la NTC 4624 "Jugos de frutas y hortalizas. Determinación del contenido de sólidos solubles. Método refractométrico". 
Comportamiento reológico: se estudió el comportamiento reológico del jugo de naranja enriquecido en sus dos concentraciones ( $1 \%$ y $5 \%)$. Se obtuvieron las curvas de flujo al aplicar un gradiente de velocidad de 2 a $100 \mathrm{~s}^{-1}$ a $25^{\circ} \mathrm{C}$. Para lo anterior se utilizó un reómetro de gradiente de velocidad controlado Anton para MCR 305 con una geometría plato-plato y un GAP (separación entre los platos) de $1 \mathrm{~mm}$. Las curvas de flujo de los jugos fueron ajustadas a los modelos de Newton o Hershel-Bulkley de acuerdo a su comportamiento.

Propiedades colorimétricas: para realizar el análisis de color de los jugos se utilizó un colorímetro Hunter Lab ColorQuest XE equipado con iluminante D65 y observador a $10^{\circ}$. Los datos obtenidos muestran las coordenadas de color $\mathrm{L}^{*}$ (luminosidad), $\mathrm{a}^{\star}$ que representa los valores rojos (positivos) y verdes (negativos) y $b^{\star}$ que representa la contribución del color amarillo (valores positivos) o azul (valores negativos) en la muestra.

\section{Resultados y discusión}

Los análisis fisicoquímicos evidenciaron mayores valores de sólidos solubles, el pH y la acidez titulable se expresó en porcentaje de ácido cítrico con un aumento de la concentración de jugo de naranja y polen. El tratamiento con mayor contenido de polen aumentó su concentración de sólidos solubles posiblemente debido a que el contenido de agua del jugo disminuyó al ser absorbida por el polen (Guillermo, 2017). La acidez titulable es un indicador de la cantidad de ácido orgánico en la muestra, se obtuvo el resultado esperado debido a que este valor aumentó con una mayor adición de jugo de naranja a la formulación.

Los tratamientos analizados cumplen con lo especificado en la NTC 5468 Jugo (zumo), pulpa, néctar de frutas y sus concentrados, tal como se muestra en la tabla 1.

Tabla 1. Propiedades fisicoquímicas del jugo de naranja enriquecido

\begin{tabular}{|c|c|c|c|}
\hline Muestra & ${ }^{\circ}$ Brix & pH & $\begin{array}{c}\text { Acidez titulable } \\
\text { (\% de ácido cítrico })\end{array}$ \\
\hline Jugo 1\% & $7,05 \pm 0,04$ & $3,54 \pm 0,006$ & $0,68 \pm 0,021$ \\
Jugo 5\% & $8,35 \pm 0,07$ & $3,8 \pm 0,01$ & $0,89 \pm 0,044$ \\
\hline
\end{tabular}

Fuente: Villa et al. 2018.

Para realizar el análisis de color de los polinéctar se utilizó un colorímetro Hunter Lab ColorQuest XE, equipado con iluminante D65 y observador a $10^{\circ}$. Todos los análisis fueron realizados por triplicado y los datos obtenidos mues- tran las coordenadas de color $\mathrm{L}^{\star}$ (luminosidad), $a^{\star}$ que representa los valores rojos (positivos) y verdes (negativos) y $\mathrm{b}^{*}$ que representa la contribución del color amarillo (valores positivos) o azul (valores negativos) en la muestra. 
En el valor de $L^{*}$, los tratamientos con adición de polen presentan mayor luminosidad (claridad o contribución del color blanco) al compararlos con la muestra comercial. La incorporación del polen influyó de manera significativa en el aumento del valor de $\mathrm{L}^{*}$, lo cual puede atribuirse a la predominancia de los granos de polen de color blanco y amarillo, lo cual varía dependiendo de su origen botánico (Guillermo, 2017).

En los valores de $b^{*}$, la muestra comercial presentó mayor contribución del color amarillo, la cual disminuyó con la adición de polen, debido, posiblemente a la presencia en mayor cantidad de los granos de color blanco (Saavedra, et al., 2013). Además, los valores altos de $b^{\star}$ en la muestra control se deben a una mayor concentración de la fruta en la formulación, lo cual podría llevar a un aumento en el contenido de luteína y zeaxantina, como pigmentos responsables del color amarillo en la pulpa de la naranja.

La contribución del color rojo o de los valores de $\mathrm{a}^{\star}$ en el color total del jugo se vio influenciada de manera positiva por la incorporación y el aumento en la concentración de polen en la formulación, en contraste con la muestra control que presentó una leve contribución del color verde en el color total, como se observa en la tabla 2.

Tabla 1. Propiedades fisicoquímicas del jugo de naranja enriquecido

\begin{tabular}{l|c|c|c}
\hline Muestra & $\mathbf{L}^{*}$ & $\mathbf{a}^{*}$ & $\mathbf{b}^{*}$ \\
\hline Comercial & $41,0834 \pm 0,02$ & $-0,041 \pm 0,01$ & $49,098 \pm 0,04$ \\
Jugo $\mathbf{1 \%}$ & $58,890 \pm 0,0055$ & $9,653 \pm 0,007$ & $44,884 \pm 0,01$ \\
Jugo $\mathbf{5 \%}$ & $57,729 \pm 0,06$ & $12,846 \pm 0,01$ & $46,294 \pm 0,03$ \\
\hline
\end{tabular}

Fuente: Villa et al. 2018.

El reograma del jugo al 1\%, mostrado en la figura 1 , evidencia un comportamiento típico de un fluido newtoniano, aun cuando la mayoría de los jugos de frutas presentan un comportamiento no newtoniano, debido a que las pulpas de frutas están formadas por partículas de diferentes formas y tamaños en disolución. Por tanto, la pulpa y la pectina son los portamiento no newtoniano propio de los jugos y néctares (Nindo, et al., 2007). El comportamiento newtoniano del jugo al $1 \%$ analizado puede estar relacionado con la concentración del mismo, debido a que en disolución este producto posee una baja concentración de fruta $y$, en consecuencia, el comportamiento newtoniano exhibido puede atribuirse al agua como componente del jugo. 


\section{REOGRAMAJUGO 1\%}

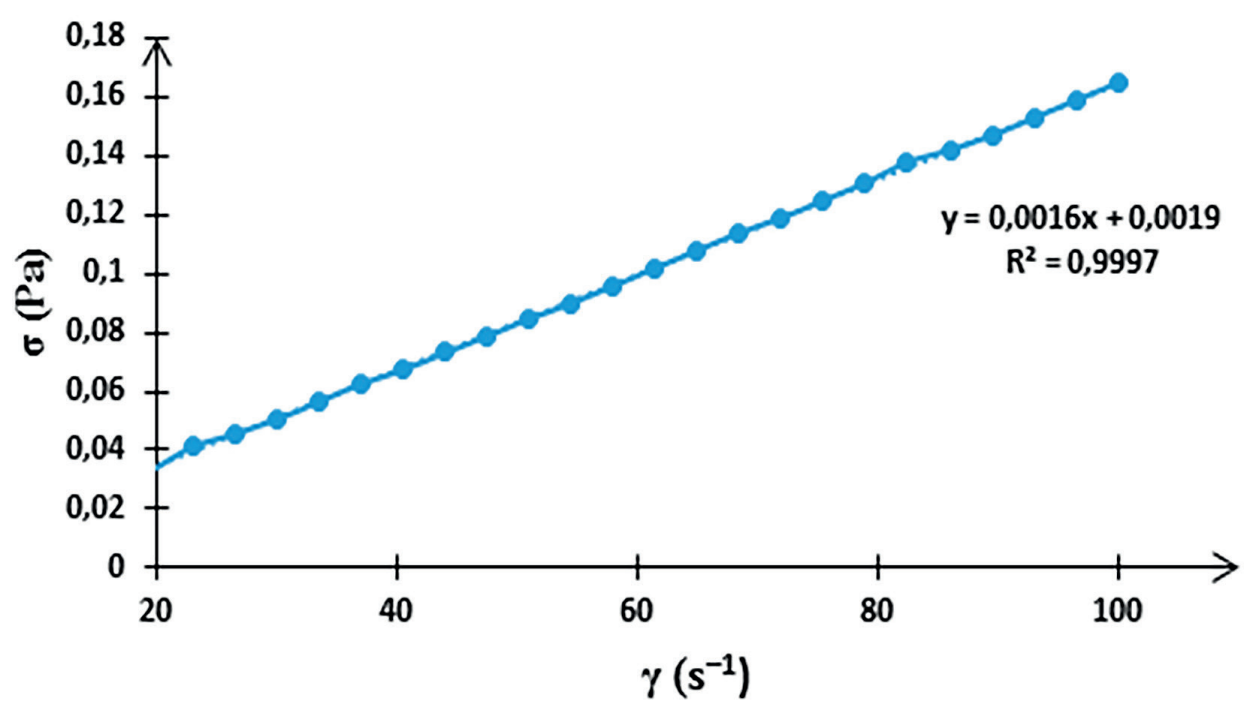

Figura 1. Reograma del jugo de naranja enriquecido con polen apícola al $1 \%$ Fuente: Villa et al. 2018.

Como se puede observar en las figuras 2 a y 2 b, el jugo al $5 \%$ muestra un comportamiento pseudoplástico, propio de los jugos y néctares de frutas, evidenciándose por la disminución de la viscosidad contra la velocidad de deformación $(\gamma)$. Al realizar el ajuste al modelo de Hershel-Bulkley se obtiene un valor de $\mathrm{n}=0,8425 \mathrm{y}$ un valor de $\mathrm{K}$ $=0,004$. El valor de K, denominado índice de consistencia, del jugo al $5 \%$ es bajo y su componente mayoritario, al igual que en el jugo al $1 \%$, es el agua; sin embargo, se evidencia el efecto de la composición del jugo en su comportamiento de flujo, debido al aumento de la cantidad de sólidos en el sistema (carbohidratos y proteínas). Se ha reportado que los purés de fruta se comportan como no-newtonianos, como resultado de una compleja interacción entre los azúcares solubles, las sustancias pécticas y los sólidos suspendidos (Ahmed, et al., 2004). 


\section{REOGRÁMA JUGO 5\%}

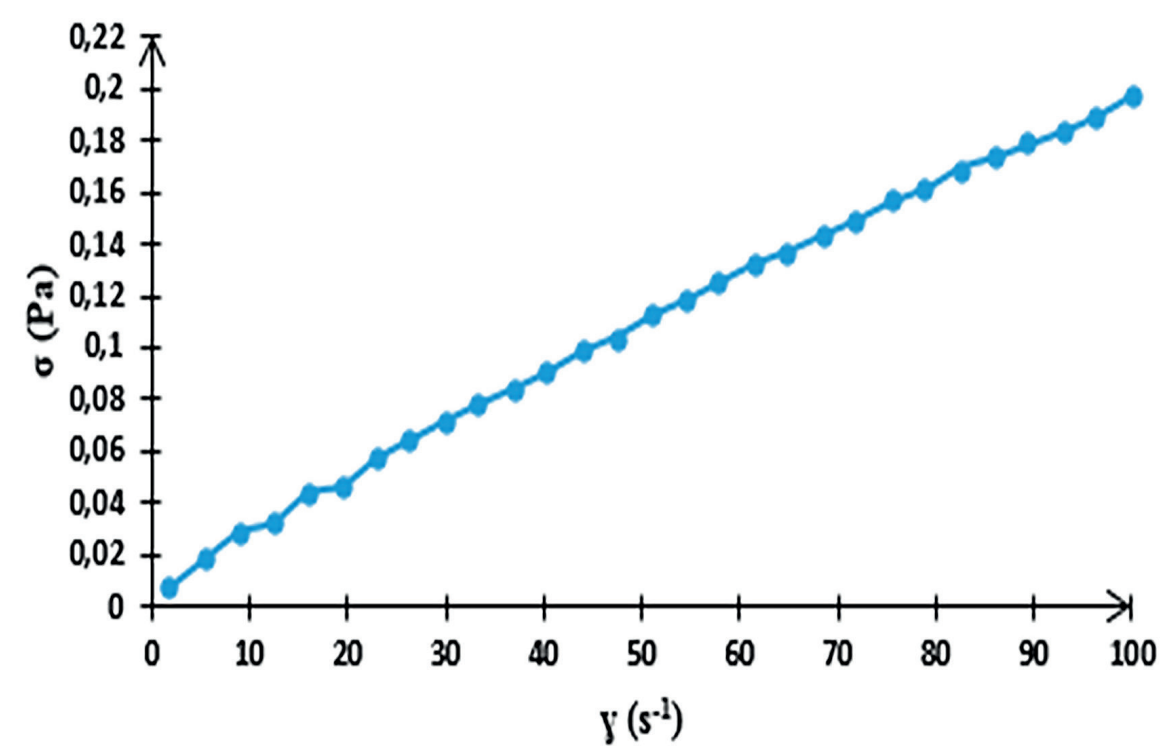

Figura 2a. Reograma del jugo de naranja enriquecido con polen apícola enriquecido al $5 \%$ Fuente: Villa et al. 2018.

\section{VISCOSIDAD JUGO 5\%}

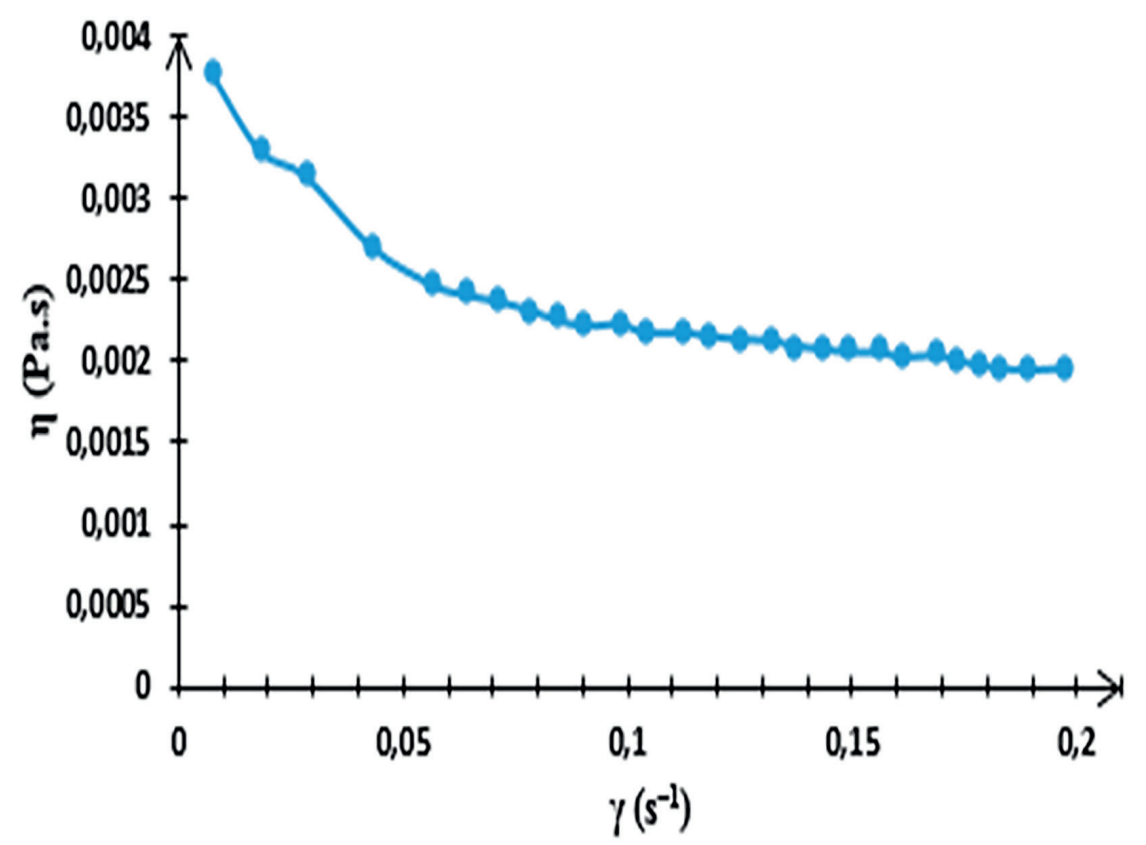


La pectina y la cantidad de partículas dispersas determinan las propiedades del flujo de las pulpas. El comportamiento pseudoplástico exhibido por el jugo al $5 \%$ puede ser explicado por la ruptura de una estructura de moléculas de polisacáridos durante el cizallamiento, tal y como describen Morris, et al. (1981) y Bhandari, et al. (2002). En este caso, la velocidad de ruptura de las interacciones moleculares existentes es superior a la velocidad de reestructuración de las mismas con el aumento de la velocidad de deformación, como resultado se obtiene una menor resistencia intermolecular al flujo y, por tanto, una disminución en la viscosidad, comportamiento presentado por el jugo al $5 \%$.

\section{Conclusión}

El aumento en la concentración de polen en el jugo de naranja tuvo un efecto significativo en las propiedades reológicas, donde se pudo evidenciar que dicho aumento en la concentración cambia las propiedades reológicas del fluido, al pasar de un fluido newtoniano para el jugo al $1 \%$ hasta un fluido no newtoniano de tipo pseudoplástico en el jugo al $5 \%$, debido principalmente al efecto de la de los carbohidratos y de la proteína en la estructura del fluido.

La adición y el aumento de concentración del polen produjo un aumento en la luminosidad del fluido, así como una mayor contribución del color rojo en el jugo, formulado gracias a las tonalidades propias de los granos de polen apícola.

\section{Agradecimientos}

Facultad de Ciencias Agroindustriales de la Universidad del Quindío. 


\section{Referencias bibliográficas}

Ahmed, J., Shivare, U. \& Singh, P. (2004). Colour kinetics and rheology of coriander leaf puree and storage characteristics of the paste. Food Chemistry, 84, pp. 605-611.

Algarra, E. (2017). Viscosidad del zumo obtenido por rehidratación de naranja en polvo en función de su tamaño de partícula. (Trabajo de grado en ciencia y tecnología de alimentos). Universidad Politécnica de Valencia, Valencia, España.

Barbosa, G., Kokini, J., Ma, L. \& Ibarz, A. (1996). The rheology of semiliquid foods. Adv. Food Nutr. Res., 39, pp. 1-69.

Benedetti, L. y Pieralli, L. (1990). Apicultura: El individuo, la colectividad, el medio, los productos de la colmena, constitución y manejo del colmenar, apicultura especializada, adversidad y medidas de prevención, legislación apícola española. Barcelona: Editorial Omega.

Bhandari, P., Singhal, R. \& Kale, D. (2002). Effect of succinylation on the rheological profile of starch pastes. Carbohydrate Polymers, 47, pp. 365-371.

Gonzáles, O. (2011). Nutrición consciente. Vitalidad y bienestar por la alimentación. Valencia, España: Ediciones Vida sana.

Guillermo, B. (2017). Efecto del contenido de miel y polen en las características físico-químicas y sensoriales del almíbar de mango (Mangífera indica). (Proyecto para optar por el título de ingeniero en agroindustria alimentaria). Escuela Agrícola Panamericana, Zamora, Honduras.
Instituto Colombiano de Normas Técnicas. (1999). NTC 4623 Productos de frutas y verduras. Determinación de la acidez titulable. Bogotá, Colombia: NTC.

Instituto Colombiano de Normas Técnicas. (1999). NTC 4592 Productos de frutas y verduras. Determinación del pH. Bogotá, Colombia: NTC.

Instituto Colombiano de Normas Técnicas. (2012). 4624 Jugos de frutas y hortalizas. Determinación del contenido de sólidos solubles. Método refractométrico. Bogotá, Colombia: NTC.

Instituto Colombiano de Normas Técnicas. (2012). NTC 5468 Jugo (zumo), pulpa, néctar de frutas y sus concentrados. Bogotá, Colombia: NTC.

Morris, E., Cutler, A., Ross, S., Rees, D. \& Price, J. (1981). Concentration and shear-rate dependence of viscosity in random coil polysaccharide solutions. Carbohydrate Polymers, 1 , pp. 5-21.

Nindo, C., Tang, J., Powers, J. \& Takhar, P. (2007). Rheological properties of blueberry puree for processing applications. LWT - Food Science and Technology, 40, pp. 292-299.

Saavedra, K., Rojas, C. y Delgado, G. (2013). Características polínicas y composición química del polen apícola colectado en Cayaltí (Lambayeque - Perú). Revista Chilena de Nutrición, 40(1), pp. 71-78. Recuperado de http:// www.scielo.cl/scielo.php?pi$\mathrm{d}=$ S0717-75182013000100011\&script=sci_arttext 